Ökonomische und politische Rahmenbedingungen für eine nachhaltige Landwirtschaft

\title{
Die Politik ist gefordert
}

\author{
Nachhaltigkeit erfordert, auch externe und intergenerativ wirksame Effekte in \\ wirtschaftliches Handeln einzubeziehen. In der Landwirtschaft finden derzeit \\ allerdings weder ihre negativen noch ihre positiven Externalitäten hinreichend \\ Berücksichtigung. Um den Strukturwandel der Landwirtschaft in eine nachhalti- \\ ge Richtung zu lenken, ist insbesondere der politische Rahmen zu verändern.
}

D Von Siegfried Bauer e seit langem bekannte Erkenntnis, dass eine Steigerung des Sozialprodukts im Sinne der volkswirtschaftlichen Gesamtrechnung nicht eine Zunahme der Wohlfahrt bedeutet, hat sich mittlerweile auf breiter Ebene durchgesetzt. Nachhaltige Entwicklungskonzepte sollen deshalb insbesondere auch qualitative Aspekte, etwa durch Sozial- und Umweltindikatoren, einbeziehen. Aus theoretisch-methodischer Sicht lassen sich normative Vorstellungen über nachhaltige Strukturen durch eine langfristige Optimierung der Wirtschaftsweise bezïglich des gesamtwirtschaftlichen Wohlfahrtsziels gewinnen (unter Einschluss privater und öffentlicher Güter und Berücksichtigung externer Effekte). Im Umkehrschluss bedeutet dies, dass die Bedingungen der Nachhaltigkeit bei kurzfristiger Optimierung und bei Vorliegen externer und intergenerativ bedeutsamer Nebenwirkungen der Wirtschaftsweise nicht eingehalten werden. Im allgemeinen hat sich heute die Definition durchgesetzt, dass eine nachhaltige Entwicklung ökologische, ökonomische und soziale Zielsetzungen im einem ausgewogenen Verhältnis berücksichtigen muss.

Die ökologische Dimension zielt auf die Belastung der Umweltmedien und die Erhaltung ihrer Qualität im Zeitablauf ab. Dabei soll die Belastung nicht größer sein als der Abbau stofflicher Belastungen und die Regenerationsfähigkeit und Selbstreinigungskraft des Ökosystems. Gerade aus landwirtschaftlicher Sicht kann auf traditionelle Vorstellungen und Konzepte zurückgegriffen werden (zum Beispiel die Aufrechterhaltung der Bodenfruchtbarkeit), die im Kontext der aktuellen Diskussion über Nachhaltigkeit weiter konkretisiert und verallgemeinert auf andere Bereiche übertragen werden.

Der soziale Aspekt von Nachhaltigkeit bezieht sich auf die Betroffenheit sozialer Gruppen und die Teilnahme einzelner Gruppen an der wirt-

schaftlichen und sozialen Entwicklung: Projekte und Politikmaßnahmen, die einseitig bestimmte Gruppen bevorzugen, erfüllen im Allgemeinen nicht die Bedingungen der Nachhaltigkeit. Wenn zum Beispiel durch eine bestimmte Politik beziehungsweise durch ein Projekt soziale Spannungen entstehen, kann der langfristige Erfolg gefährdet sein.

Ökonomische Nachbaltigkeit eines Projektes oder eines politischen Konzepts meint dessen Wirtschaftlichkeit. Bezogen auf die Landwirtschaft werden darunter Konstellationen verstanden, die eine Überlebensfähigkeit der Landwirtschaft ermöglichen. Dazu ist vor allem eine ausreichende Rentabilität und Liquidität der Landbewirtschafter erforderlich. Ökonomische Nachhaltigkeit im Sinne der Erhaltung des Unternehmens und des unternehmerischen Wachstums erfordert dabei vor allem eine positive Eigenkapitalbildung. Umgekehrt ausgedrückt ist bei einem dauerhaft steigenden Verschuldungsgrad die nachhaltige Entwicklung gefährdet. Dieses unternehmerische Nachhal-

\section{Ökoeffiziente Dienstleistungen}

und

\section{Integrierte Produktpolitik}

sind die Themen der Informationsdienste Ökologisches Wirtschaften $5 / 00$ und $6 / 00$

Wenn Sie potenzielle Beiträge haben, wenden Sie sich bitte an die Redaktion. tigkeitspostulat spielt gerade in der Landwirtschaft eine zentrale Rolle (Weitergabe des Betriebs an die nachfolgende Generation).

\section{- Strukfurwandel der Landwirt- schaft und Nachhaltigkeit}

Betrachtet man den permanenten Strukturwandel der Landwirtschaft, so wird deutlich, dass Nachhaltigkeit in der Landwirtschaft nicht im Sinne eines Fortbestehens aller derzeitigen landwirtschaftlichen Betriebe interpretiert werden kann. Im Zuge des landwirtschaftlichen Strukturwandels ist bei begrenzter Fläche ein Ausscheiden von Betrieben eine notwendige Voraussetzung für das Wachstum anderer Betriebe. In den vergangenen zwei Jahrzehnten verminderte sich die Zahl der landwirtschaftlichen Betriebe um etwa drei bis vier Prozent jährlich. Diese Tendenz dürfte auch in der Zukunft anhalten, da die wesentlichen Bestimmungsfaktoren auch künftig wirksam sein werden, insbesondere:

- begrenztes Wachstum der Nachfrage nach Nahrungsmitteln (Engel'sches Gesetz),

- vergleichsweise hohe Raten des biologischund mechanisch-technischen Fortschritts,

- Druck auf die Realisierung von Fortschritten durch die steigende Lohn-Zins-Relation und die steigenden gesamtwirtschaftlichen Einkommen,

- Auslagerung von Funktionen aus der Landwirtschaft in spezialisierte Unternehmen im vorund nachgelagerten Bereich.

Die Fortsetzung des bisherigen Strukturwandels ist daher eine Voraussetzung für eine ökonomisch nachhaltige Landwirtschaft. Ökonomische Nachhaltigkeit im Sinne eines dauerhaften Bestehens kann daher nur für einen Teil der landwirtschaftlichen Betriebe gelten.

Auch aus ökologischer Sicht muss Interesse an einem fortschreitenden Strukturwandel bestehen, damit überhaupt noch überlebensfähige Betriebe aufrecht erhalten beziehungsweise geschaffen werden können. Wenn sich die Nutzung betriebswirtschaftlich nicht rechnet und einen adäquaten Lebensstandard nicht länger gewährleisten kann, ist in vielen Regionen die Agrarnutzung und damit die Nachhaltigkeit der Landwirtschaft gefährdet.

Nachhaltige Landwirtschaft muss folglich über die einzelbetriebliche Sicht hinaus aus regionaler Perspektive, das heißt im Sinne der Aufrechterhaltung einer hinreichenden Zahl überlebens- und entwicklungsfähiger Betriebe betrachtet werden. Auch unter sozialen Ge- 
sichtspunkten ist ein weiterer Strukturwandel notwendig, um den verbleibenden Menschen in der Landwirtschaft einen adäquaten Lebensstandard zu sichern.

\section{- Positive und negative externe Effekte}

Auf der einzelbetrieblichen Entscheidungsebene besteht in Teilbereichen ein gewisses Eigeninteresse, ökologische und ökonomische Gesichtspunkte gleichzeitig zu berücksichtigen, um die derzeitigen und künftigen Produktionsgrundlagen zu sichern, wie zum Beispiel die Aufrechterhaltung der Bodenfruchtbarkeit, Erosionsschutzmaßnahmen oder die geringe Schadstoffbelastung von Boden, Wasser und Luft. Dem steht allerdings gegenüber, dass Unternehmen in einer Marktwirtschaft häufig kurzfristig einem starken Wettbewerbsdruck ausgesetzt sind, der dazu führen kann, dass langfristige Aspekte und ökologische Kriterien vernachlässigt werden, um ökonomisch zu überleben.

Auf der anderen Seite bestehen eine Reihe echter Externalitäten, die bei gegebenen ökonomischen Rahmenbedingungen und den einzelwirtschaftlich ausgerichteten Verhaltensweisen landwirtschaftlicher Unternehmer (marktwirtschaftliche Strukturen und Entscheidungsprozesse) zu Belastungen der Umweltmedien fuihren. Beispiele für solche negativen externen Effekte (Umweltbelastungen) der Landwirtschaft sind:

- Grundwasserverschmutzung und Belastung von Fließgewässern,

- Verlust der Bodenfruchtbarkeit (Verdichtung und Erosion),

- Verminderung der Artenvielfalt (Biotopbelastung und -vernichtung),

- Beitrag zum Treibhauseffekt durch Kohlendioxid- und Methanemissionen.

Nicht zu vernachlässigen sind in diesem Funktionsgefüge die Nettobelastungen durch die wirtschaftlichen Aktivitäten in Ballungsgebieten, die ein Gefährdungspotenzial für die Landwirtschaft darstellen (zum Beispiel Schwermetallbelastung, Luftverschmutzung, Gewässerbelastung).

Daneben sind mit den landwirtschaftlichen Aktivitäten aber auch positive externe Effekte (Umweltleistungen) verbunden, beispielsweise:

- Offenhaltung der Landschaft (Grundwasserneubildung, Freizeit und Erholung),

- Erhalt der Artenvielfalt (Agrarökosysteme),

- Luftreinigung und Sauerstoffproduktion.

Diese positiven externen Effekte der Landwirtschaft werden ebenso wie die externen Belas-

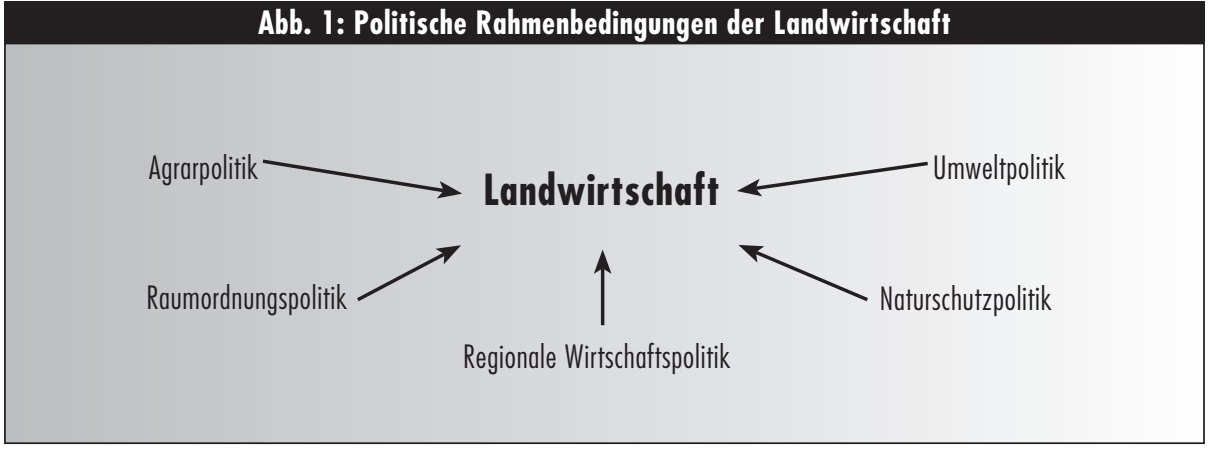

tungen bisher nicht oder nur unzureichend in Rechnung gestellt, da es sich bei den bereitgestellten Leistungen um öffentliche Güter handelt. Aufgrund der nicht vorhandenen Eigentumsrechte können die landwirtschaftlichen Umweltleistungen nicht von Marktmechanismen erfasst und folglich auch nicht honoriert werden. Eine ausreichende allgemeine Bereitstellung dieser Leistungen gerät zunehmend in Konflikt mit den Interessen der ländlichen Bevölkerung und vor allem einer modernen rationellen Landwirtschaft.

\section{Bewertung der Rahmenbedingungen}

Die derzeitigen politischen Rahmenbedingungen auf europäischer und bundesdeutscher Ebene sind nicht beziehungsweise nicht durchgängig darauf ausgerichtet, die negativen und positiven externen Effekte der Landwirtschaft zu internalisieren. Die Landwirtschaft wird dabei vorrangig von der betriebenen Agrarpolitik, aber auch von einer Reihe weiterer Politikbereiche (EU, Bund, Länder und Kommunen) beeinflusst (siehe Abb. 1) (1).

Da eine einheitliche Grundausrichtung der verschiedenen Politikbereiche weitgehend fehlt, ergeben sich Widersprüche, Inkonsistenzen und Koordinationsdefizite zwischen und teilweise auch innerhalb der einzelnen Politikbereiche. Anschauliche Beispiele sind die gleichzeitige Subventionierung von Energieverbrauch (zum Beispiel Gasölbeihilfe) und von energiesparenden Maßnahmen oder die parallele Förderung der Flächenstillegung und der Aufrechterhaltung der Landwirtschaft (Bergbauernprogramm). Diese Defizite sind vor allem auf allgemeine politökonomische Kalküle der politischen Entscheidungsträger und auf eine überwiegend sektorale (und zum Teil sogar einzelwirtschaftlich verengte) Klientelorientierung zurückzuführen. Anstatt die Defizite im konzeptionellen Bereich zu beheben, wird durch die Schaffung ständig neuer umweltpolitischer Instrumente und Gesetze politischer Aktionismus betrieben. Die Folge ist eine zunehmend hohe Regelungsdichte speziell in der Umweltgesetzgebung mit den damit verbundenen Problemen.

Im Mittelpunkt der gemeinsamen Agrarpolitik der Europäischen Union (EU), die den wichtigsten Rahmen darstellt, stand bis Anfang der 90er Jahre eine aktive Agrarpreisstützung, um auf diese Weise der Landwirtschaft eine Teilnahme an der allgemeinen Einkommensentwicklung zu ermöglichen. Diese Politik war jedoch mit immer stärkeren Produktionsüberschüssen, Haushaltsbelastungen und Konflikten mit Drittländern und Handelspartnern verbunden. Gleichzeitig hat diese Politik eine intensive Landbewirtschaftung mit entsprechenden Umweltbelastungen begünstigt. Aufgrund der Finanzprobleme und des Drucks von Drittländern erfolgte zu Beginn der 90er Jahre eine umfassende Agrarreform. Die Agrarpreise wurden zum Teil drastisch gesenkt und der Einkommensausfall über flächenbezogene Ausgleichszahlungen annähernd ausgeglichen. Gleichzeitig wurde eine obligatorische Flächenstillegung eingeführt, um kurzfristig einen entsprechenden Überschussabbau zu bewirken. Zusätzlich zu diesen marktbezogenen Maßnahmen wurden sogenannte flankierende Maßnahmen eingeführt, die auf freiwilliger Basis eine stärker umweltschonende Landbewirtschaftung anregen sollten. Im Ergebnis hat diese Politik zwar auf einer Reihe von Märkten den Produktionsanstieg gedämpft, eine grundlegende Umkehr der Agrarentwicklung und eine durchgängig stärkere Berücksichtigung von Umweltbelangen im Sinne von Nachhaltigkeit trat jedoch nicht ein.

Die anhaltende Problemlage hat dann im Jahre 1999 zur Agenda 2000 der EU geführt, die im wesentlichen den Kurs von 1992 weiter betrieben hat. Es erfolgte eine weitere Senkung der Agrarpreise und eine Aufstockung der Flächen- 
und Tierprämien als Kompensation für die Agrarpreissenkung. Die flankierenden Maßnahmen wurden zusammen mit den Maßnahmen zur Agrarstrukturförderung in eine neues Paket von Maßnahmen zur ,Förderung der ländlichen Entwicklung“ gegossen. Mit der Agenda 2000 war die Hoffnung verbunden, die agrarpolitischen und finanziellen Voraussetzungen für die Osterweiterung der EU sowie für die anstehenden Liberalisierungsverhandlungen im Rahmen der Welthandelsorganisation (WTO) zu schaffen. Inwieweit sich die beiden Hoffnungen erfuillen und als hinreichende Voraussetzungen für die beiden globalen Veränderungen gelten können, bleibt vorerst abzuwarten.

Die aus den Politikänderungen resultierenden Umweltwirkungen sind bestenfalls als korrigierend und reparierend, nicht aber zielgerecht steuernd anzusehen.

\section{- Nachhaltige Lösungsansätze}

Als Konsequenz aus den bisher dargestellten Sachverhalten wurde im Hinblick auf eine nachhaltige Landbewirtschaftung von verschiedenen Seiten ein stärker marktwirtschaftliche Instrumentenmix vorgeschlagen (2).

\section{Dökostevern}

Unter Ökosteuern wird hier eine schrittweise Besteuerung fossiler Energieträger sowie umweltbelastender Emissionen und landwirtschaftlicher Betriebsmittel verstanden (zum Beispiel Mineraldünger, Pflanzenschutzmittel). Der entscheidende Vorteil in der Anwendung von Ökosteuern besteht in der durchgängigen Wirkung über alle Sektoren, Regionen und Produktionsketten hinweg (Preisüberwälzungseffekte).

Neben der zu erwartenden flächendeckenden Senkung der Umweltbelastungen ist durch die Einführung einer Ökosteuer vor allem mit wegweisenden Impulsen zum Rückbau der hohen umweltrechtlichen Regelungsdichte zu rechnen. Langfristig dürften viele Umweltprobleme aus der umweltrechtlichen Regelung und Kontrolle herausfallen.

\section{- Honorierung ökologischer Leistungen}

Ein System zur Honorierung ökologischer Leistungen der Landwirtschaft stellt das konsequente Gegenstiick zur steuerlichen Sanktionierung negativer Umweltwirkungen dar. Anstelle von Geboten und Verboten werden marktwirtschaftliche Anreize zur Erbringung von Umweltleistun- gen gesetzt. Dabei sollen die positiven externen Effekte spezieller Bodennutzungsformen und Betriebssysteme, die sich durch verringerte Intensität, vielfältigere Fruchtfolgen und einem höheren Anteil von Landschaftsstrukturelementen auszeichnen, unter Berücksichtigung der regionalen Knappheiten (und damit der gesellschaftlichen Präferenzen) durch finanzielle Anreize gefördert werden. Wünschenswert ist ein outputorientierter Ansatz, der, im Gegensatz zu den bisherigen input- und handlungsorientierten Konzepten der Agrar- und Umweltpolitik, verstärkt die ökologischen Effekte und somit die tatsächlich erbrachten ökologischen Leistungen berücksichtigt.

\section{- Ökologisch erweiterter Finanzausgleich}

Analog zum Honorierungsansatz für landwirtschaftliche Unternehmen ist eine Erweiterung des bestehenden kommunalen Finanzausgleichs darauf ausgerichtet, überregional bedeutsame Umweltleistungen und -belastungen (positive und negative Spillovers) zu internalisieren, indem übergeordnete staatliche Ebenen (Bund, Länder) als Nachfrager und Kommunen als Anbieter von Umweltleistungen fungieren. Dadurch werden für öffentliche Entscheidungsträger Anreize zu einer umweltgerechten Kommunal- und Regionalentwicklung geschaffen. In einem solchen System würden etwa einer Stadt, die durch Flächenversiegelung oder Luftverschmutzung ihre Umgebung besonders belastet, die Zuweisungen gekürzt. Andererseits werden umweltund naturverträgliche Aktivitäten wie die Ausweisung von Natur- oder Wasserschutzgebieten, welche die Entwicklungsmöglichkeiten einer Kommune beschränken, finanziell entlohnt. In einem solchen System wäre auch eine zielspezifische Förderung der Landwirtschaft auf kommunaler Ebene möglich.

\section{Ausblick}

Eine Politik für eine nachhaltige Entwicklung der Landwirtschaft sollte nicht nur an den Symptomen im Sinne nachträglicher Reparaturen ausgerichtet werden, sondern muss bei den eigentlichen Ursachen von Problemen ansetzen. Diese Ursachen liegen vorrangig bei den politischen und wirtschaftlichen Rahmenbedingungen, welche die wirtschaftlichen Aktivitäten der Landwirtschaft steuern. Gegenüber der bisher mehr additiven Agrar-, Regional- und Umweltpolitik mit einer überwiegend partiellen Aus- richtung auf nachträgliche Teilreparaturen und ihren inhärenten Widersprüchen, ist mehr denn je eine stärker integrierende Politikgestaltung und eine Durchforstung und ein Rückbau des derzeitigen Steuer- und Subventionssystems notwendig.

Die Vorschläge zur Honorierung ökologischer Leistungen sind bislang von der Politik, auch in den Beschlüssen zur Agenda 2000, kaum aufgegriffen worden. Möglicherweise werden umweltleistungsorientierte Honorierungskonzepte an Bedeutung gewinnen, wenn die Probleme der pauschalen, nichtleistungsbezogenen Flächenbeihilfen im Rahmen der WTO-Verhandlungen sich als nicht tragfähig und die Finanzierungsprobleme durch die anstehende Osterweiterung der Gemeinschaft sich als kaum lösbar herausstellen.

\section{Anmerkungen}

(1) Vgl. Bauer, S.: Naturschutz und Agrarpolitik. In: Konold, W./ Böcker, R./ Hampicke, U. (Hrsg.): Handbuch Naturschutz und Landschaftspflege, Landsberg 1997.

(2) Vgl. Bauer, S./ Abresch, J.P./ Steuernagel, M.:

Gesamtinstrumentarium zur Erreichung einer umweltverträglichen Raumnutzung. Materialien zur Umwelfforschung, $\mathrm{Nr}$. 26, Stuttgart 1996; Rat von Sachverständigen für Umweltfragen: Umweltgutachten: Für eine dauerhaft-umweltgerechte Entwicklung, Stuttgart 1994; Streit, M./ Wildemann, R./ Jesinghaus, J.: Landwirtschaft und Umwelt. Wege aus der Krise, Baden-Baden 1989; Mayrhofer, P.: Modell Ökopunkte Landwirtschaft. Der Ökopunkteschlüssel, Verein zur Förderung der Landentwicklung und intakter Lebensräume (Hrsg.), Basel 1992.

(3) Vgl. Bauer, S.: Honorierung ökologischer Leistungen der Landwirtschaft: Was hat sich in den letzten Jahren getan? Was ist in nächster Zeit zu erwarten? In: Jarre, J. (Hrsg.): Landwirtschaftliche Identität zwischen Unternehmertum und staatlichen Transferleistungen. Loccumer Protokolle 6/99, 1999, S. 107-132.

\section{Der Autor}

Dr. Siegfried Bauer ist Professor für Projekt- und Regionalplanung an der Universität Gießen. Kontakt: Fachbereich Agrarwissenschaften und Umweltsicherung an der Justus-Liebig-Universität Gießen, Diezstraße 15, 35398 Gießen, Tel. 0641/ 99373-10, Fax -19, E-mail: Siegfried.Bauer@agrar.uni-giessen.de 
(c) 20I0 Authors; licensee IÖW and oekom verlag. This is an article distributed under the terms of the Creative Commons Attribution Non-Commercial No Derivates License (http://creativecommons.org/licenses/by-nc-nd/3.o/), which permits unrestricted use, distribution, and reproduction in any medium, provided the original work is properly cited. 